\title{
Associations de lutte contre le cancer et politisation : quels rôles des patients?
}

Sandrine Knobé

L'expression collective de doléances propres aux usagers de la santé n'émerge pas subitement dans les années 1980-1990 avec les actions associatives très médiatisées comme celles de la lutte contre le sida (Pinell, 2002 ; Dodier, 2003). Mais cette période s'avère toutefois propice à une politisation progressive de la question de la participation des usagers au processus d'élaboration des politiques publiques de santé du fait, entre autres, de la médiatisation de différentes affaires dites de santé publique (Mougeot et al., 2018). Les années 1990 voient fleurir dans les médias un certain nombre de scandales sanitaires qui, à ce moment-là, seront publicisés comme de graves crises de santé publique où les victimes ellesmêmes montent au créneau (Vilain, Lemieux, 1998 ; Barbot, Fillion, 2007). Le drame du sang contaminé (Fillion, 2009), qui en représente l'exemple paradigmatique, a fortement marqué l'opinion publique ainsi que les acteurs politiques eux-mêmes dans la mise en cause de responsabilités au plus haut niveau de l'État.

Tout au long des années 1990, d'autres affaires vont tour à tour connaître une publicisation importante, portées ou non par des collectifs porte-parole de victimes ${ }^{1}$, parmi lesquelles les affaires relatives à l'hormone de croissance, à l'amiante (Henry, 2000), à la vache folle (Hirsch et al., 1996) ou à la pollution de La Hague (Veil, 1998). La multiplication de ces «affaires » mais surtout leur médiatisation et la mise en exergue d'usagers victimes participent à créer des conditions sociales favorables à la naissance et à la réussite de mouvements contestataires portés par des usagers eux-mêmes ou leurs représentants. Le spectre de «crise de santé publique » incite les pouvoirs publics à commanditer des rapports d'informations ${ }^{2}$. À l'occasion de telles enquêtes publiques, les associations de victimes ou représentant les usagers sont en général auditionnées afin d'exposer leurs principales revendications.

\section{Des patients, acteurs des politiques publiques}

La thématique de la participation des usagers au système de soins s'insère ainsi dans le débat politique et connaît plusieurs traductions juridiques, jusqu'à devenir un enjeu reconnu sous l'appellation de "démocratie sanitaire» (Tabuteau, 2007, 2013). Cet enjeu traverse également le domaine de la lutte contre le cancer. Ainsi, les premiers «États généraux des malades atteints de cancer », qui rassemblent environ 1300 malades, sont organisés par la Ligue nationale contre le cancer (LNCC) à Paris en novembre 1998 (Ligue, 1999).

\footnotetext{
${ }^{1}$ Comme par exemple : Association de défense des victimes de l'affaire du sang contaminé (ADVASC) créée en 1995 ; Association nationale de défense des victimes de l'amiante (ANDEVA) créée en 1996; Association des victimes de la maladie de Creutzfeldt-Jakob (AVMCJ) créée en 1997 ; Association des victimes de l'hormone de croissance (AVHC) créée en 1999.

${ }^{2}$ Exemples : rapport Mattei (1997), Sur l'ensemble des problèmes posés par le développement de l'épidémie d'encéphalopathie spongiforme bovine ; rapport Revol et Le Déaut (1997), L'amiante dans l'environnement de l'homme : ses conséquences et son avenir.
} 
L'événement sera perçu comme un acte fondateur de la prise de parole des malades par les acteurs de la lutte contre le cancer et de son institutionnalisation (Briatte, Knobé, 2010). Certaines revendications, comme celle relative à l'instauration d'un « dispositif d'annonce », seront reprises lors du premier «Plan Cancer» (2003-2007) (Bevetty et al., 2006).

Durant les années 1990, se créent d'autres associations qui se positionnent clairement du côté d'un activisme politique en faveur des patients (comme par exemple Europa Donna Forum France ou Jeunes Solidarité Cancer). Ces associations cherchent à défendre les intérêts de leurs adhérents en militant notamment pour une prévention précoce, un dépistage organisé, un accès aux meilleurs traitements, autant de revendications qui rappellent l'activisme thérapeutique mis en avant par les associations de lutte contre le sida (Barbot, 2002). Elles développent aussi des actions sur le plan social pour une meilleure (ré)insertion professionnelle des patients par exemple. Cet activisme dans le domaine du cancer est plus tardif et plus modéré qu'aux États-Unis où les collectifs se mobilisent dès les années 1970 pour faire pression sur les acteurs politiques afin que des mesures concrètes soient prises en matière de prévention, de soin ou de recherche (Ménoret, 2002, 2006 ; Gaudillière, 2002 ; Derbez, Rollin, 2016).

\section{L'obstacle des expériences personnelles}

Toutefois, dans ces associations, l'activisme politique se heurte à un «entre-soi » des patients difficile à dépasser (Knobé, 2015). L'expérience personnelle de la maladie ne se transforme en cause collective qu'à la suite d'un travail associatif visant à transformer des « causes particulières » en «causes générales » (Pollak, 1991). Les principaux acteurs de cette transformation ne sont pas nécessairement les patients eux-mêmes. Cette difficulté provient notamment des attentes des patients aux différents moments de la trajectoire de la maladie du cancer (Ménoret, 1999). D'une part, en début de trajectoire ou en cas de récidive, un régime de proximité directe avec la maladie encourage les attentes en termes d'entraide; d'autre part, en fin de trajectoire (ascendante), une forme d'éloignement de la maladie participe à une prise de distance ; ce qui dans les deux cas rend difficile une publicisation collective (Herzlich, 1995). La trajectoire de la maladie influe sur les attentes relatives à l'engagement associatif (Knobé, 2007 ; Ferrand-Bechmann, 2011).

La question du rôle de l'environnement associatif dans la politisation des individus a déjà fait l'objet d'études contrastées. Les associations pourraient représenter des lieux d'évitement du politique et de fixation sur les problèmes concrets et immédiats que l'association se propose de résoudre au quotidien (Eliasoph, 1998) ou au contraire constituer des lieux de socialisation propices à la politisation (Mayer, 2000). Qu'en est-il de ces associations se positionnant comme militantes ? Nous souhaiterions analyser, à l'échelle locale de certaines associations, la politisation des discours des patients. Notre attention portera sur les processus de montée en généralité des discours et de reconnaissance par les acteurs de la dimension potentiellement conflictuelle de leur prise de position (Duchesne, Haegel, 2001 ; 2004), tout en tenant compte de l'importance des interactions entre les membres des associations (Hamidi, 2006).

Notre ambition sera d'apporter des éléments de compréhension des freins à la politisation des discours et des actions des patients atteints de cancer dans le milieu associatif à travers l'analyse des logiques sociales d'engagement associatif des patients (Knobé, 2011, 2015). Mais comprendre les rôles pris par les patients dans l'espace associatif de lutte contre le cancer suppose de saisir les configurations particulières qui le caractérise. Un détour par l'histoire et l'évolution des associations de ce domaine en France constitue ainsi un préalable. Notre argumentation s'articulera autour de trois points complémentaires. Dans un premier temps, nous présenterons les principales lignes de transformation de l'espace associatif de 
lutte contre le cancer, explicatives pour une part des rôles dévolus aux patients eux-mêmes. Dans un second temps, nous expliciterons les logiques sociales d'engagement associatif des patients atteints de cancer. Puis, nous terminerons par montrer que la valorisation prédominante ou exclusive des singularités individuelles freine la politisation des discours des patients eux-mêmes, alors que pourtant l'on peut observer dans les années récentes l'institutionnalisation d'une forme légitime de représentation des usagers.

\section{Transformations de l'espace associatif de lutte contre le cancer (1996-2015)}

L'histoire, en France, du mouvement associatif dans le domaine de la santé connaît trois grandes périodes (Pinell, 1997). La première, qui correspond au 19ème siècle, est celle de la charité à l'égard de certaines catégories d'indigents malades ne trouvant leur place ni à l'hôpital ni à l'hospice. La deuxième période, qui couvre la fin du XIX et le début du XX siècle, inscrit la lutte contre la maladie dans celle contre les fléaux sociaux, et voit ainsi apparaître les « ligues » contre la tuberculose, la syphilis, l'alcoolisme ou le cancer. La Ligue contre le cancer naît le 14 mars 1918 à Paris. Enfin, la troisième période, qui démarre après la Seconde Guerre mondiale, se caractérise par une plus grande diversité des logiques d'action : collecte de fonds destinés à la recherche biomédicale (exemple de l'ARC - Association pour la recherche sur le cancer - créée en 1958), gestion de structures de prise en charge, et autoorganisation des usagers sur le modèle des groupes de self-help développés aux États-Unis (exemple de Vivre comme avant créée en France en 1975).

Graphique 1 : nombre d'associations de lutte contre le cancer créées en France entre 1996 et 2015

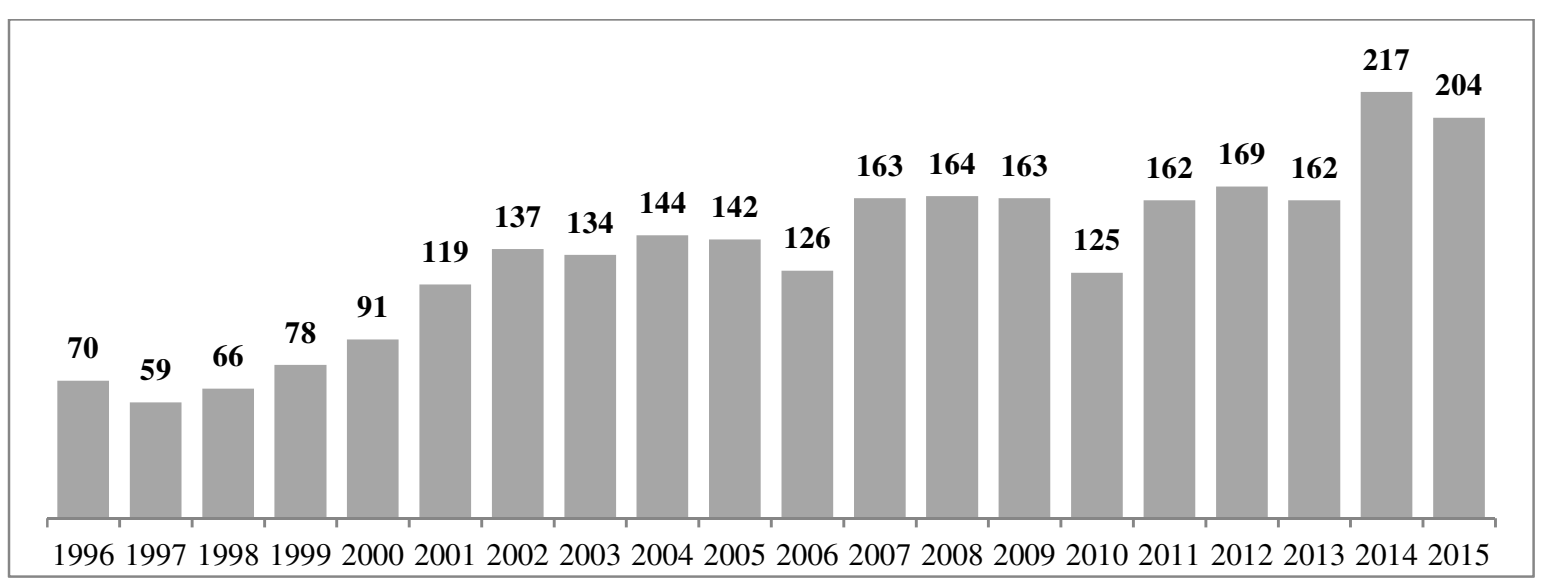

Au cours des années 1990, l'on constate également une multiplication des créations d'associations dans le domaine de la lutte contre le cancer. Le secteur associatif, tous domaines confondus, fait preuve d'une grande vitalité avec, depuis les années 1990, entre 65000 et 70000 associations créées chaque année en France dont environ $3 \%$ relèvent du domaine de la santé. Si l'on tient uniquement compte des associations de lutte contre le cancer, on observe qu'en moyenne environ 135 associations ont été créées dans cette orientation chaque année (entre 1996 et 2015) ${ }^{3}$. Depuis le milieu des années 1990, la tendance

\footnotetext{
${ }^{3}$ Pour la période 1996 à 2015 un recensement des associations de lutte contre le cancer a été effectué pour la France métropolitaine. Ont été prises en compte les déclarations de créations d'associations recensées au Journal officiel Associations loi 1901 et fondations d'entreprises dont le titre et/ ou l'objet contiennent un des mots clés suivants : cancer, cancérologie, oncologie, tumeur, chimiothérapie, radiothérapie. Base de données disponible sur Internet : http://www.journal-fficiel.gouv.fr/jahia/Jahia/associations. Les associations d'Alsace et de Lorraine
} 
est même à l'augmentation progressive du nombre annuel d'associations créées (graphique 1). L'ensemble de ces créations densifie d'année en année l'espace associatif de lutte contre le cancer. Ce nombre ne nous donne toutefois aucune indication sur la taille, l'activité effective, ou la durée de vie de ces associations. Les disparités peuvent être très grandes d'une association à l'autre et nous ne disposons malheureusement pas de données relatives à ces aspects. Cet état des lieux nous permet cependant de saisir les dynamiques de création à l'œuvre dans le domaine de la lutte contre le cancer.

Les dynamiques territoriales de création d'associations dans le domaine de la lutte contre le cancer semblent reproduire celles de l'ensemble des créations d'associations tous domaines confondus et ne pas refléter, par exemple, les disparités épidémiologiques régionales en matière d'incidence et de mortalité par cancer. Les logiques de création ne seraient peut-être pas tant liées à une incidence élevée des cas de cancers dans la région qu'à une forte « culture » associative ancrée localement. Parfois, le secteur associatif semble compenser un plus faible nombre d'établissements hospitaliers orientés en cancérologie (ou l'absence d'un Centre de lutte contre le cancer), c'est le cas par exemple pour la région Centre (cf. figure 1). À l'inverse, pour la région Ile-de-France on peut faire l'hypothèse que le nombre plus élevé d'établissements encourage les initiatives associatives.

Figure 1 : densité d'associations de lutte contre le cancer créées en France pour 100000 habitants ${ }^{4}$ entre 1996 et 2015

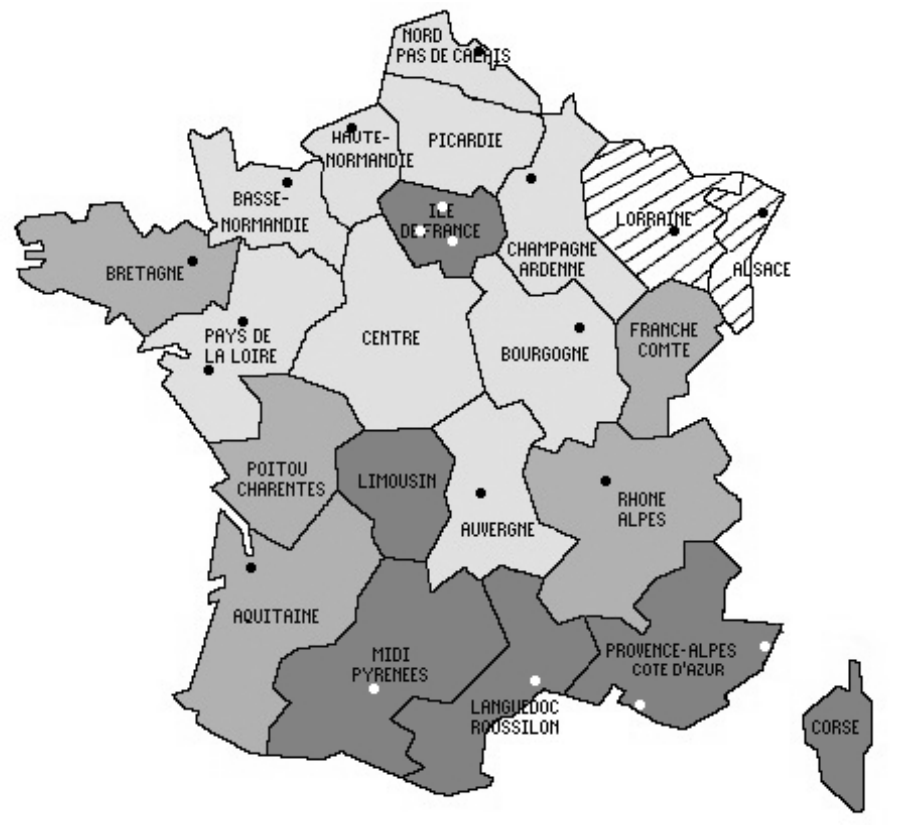

$\frac{\text { Légende : }}{\mathrm{O} \text { ou } \mathrm{CLCC}}$

Moins de 4 associations créées

De 4 à 5 associations créées

Plus de 5 associations créées

Le recensement des associations n'a pas été effectué pour l'Alsace et la Lorraine (partie hachurée)

Les domaines de la prévention, de la formation et de l'information représentent près de $30 \%$ des champs d'intervention déclarés lors de la création des associations ${ }^{5}$ (graphique 2). Des actions en faveur des malades sont envisagées par $24 \%$ des associations créées entre

n'ont pas été recensées car en Alsace et en Moselle les associations dépendent du droit local, loi 1906 et sont déclarées aux tribunaux d'instances.

${ }^{4}$ Calcul de la densité réalisé à partir des chiffres du recensement 2006.

${ }^{5}$ D'après l'objet précisé lors de la déclaration de l'association au Journal officiel Associations loi 1901. Une association peut déclarer des activités dans plusieurs domaines distincts considérés ici. La catégorie «divers » rassemble des associations proposant des actions ne s'inscrivant dans aucune des autres catégories distinguées ici. Souvent, il s'agit d'associations menant des actions de collecte de fonds destinés à d'autres associations de la lutte contre le cancer. 
1996 et 2015. La proportion d'associations proposant des actions de soutien aux malades a augmenté au fil des vingt dernières années. En effet, en 1996, seul un peu plus de $13 \%$ des associations créées ont déclaré des actions en faveur des malades. Elles sont environ $22 \%$ en 2005, et en 2015 elles représentent $24 \%$ des associations créées. À partir de 2011, c'est même ce type d'objet qui devient majoritaire.

Graphique 2 : répartition (en \%) des domaines d'action déclarés par les associations de lutte contre le cancer créées en France entre 1996 et 2015

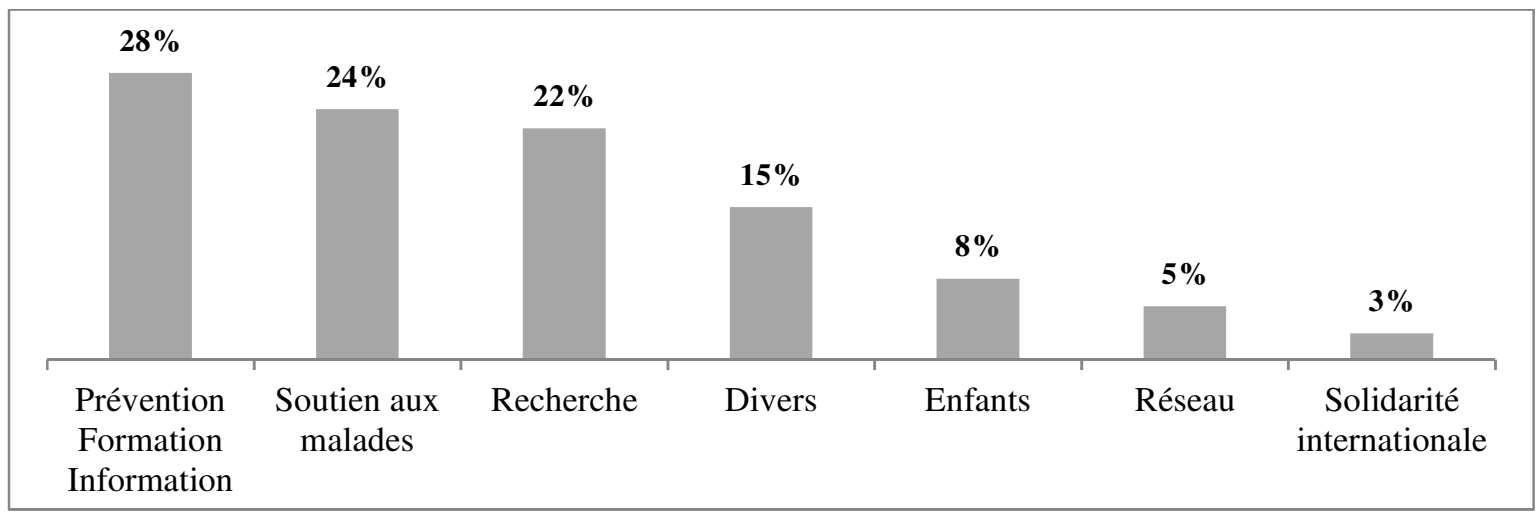

Concernant plus particulièrement les associations proposant des actions en faveur des malades les résultats d'une enquête par questionnaire ${ }^{6}$ apportent quelques indications intéressantes quant à la structuration de l'espace associatif. Le secteur des actions en faveur des malades paraît de la sorte être plus souvent investi par les patients et les proches de patients que par les membres du corps médical. Les médecins s'avèrent d'ailleurs principalement (environ $75 \%$ ) à l'initiative de la création lorsque l'association propose des activités de recherche en plus des actions en faveur des malades. Inversement, lorsque l'association ne mène pas d'activités en matière de recherche ce sont les proches et les patients qui, à $65 \%$, sont à l'origine des créations. Bien souvent, notamment dans le cas des proches, c'est l'envie d'agir en matière de lutte contre le cancer qui anime les intentions de créer des associations. La diversification relèverait surtout, dans le domaine des actions en faveur des malades, d'un investissement associatif engendré par un vécu personnel en rapport avec la maladie. Tout se passe comme si cet investissement devait permettre de faire face à un décès ou à sa propre maladie.

Quand j'ai eu mon cancer en 1999 j'ai éprouvé le besoin d'en parler avec des personnes qui avaient eu à peu près le même. Cela a été difficile à trouver. Aussi lorsque j'allais mieux j'ai demandé aux personnes rencontrées si elles voulaient que nous fassions quelque chose. Dans un premier temps nous avons été un groupe au sein d'une association, mais très vite nous avons senti qu'il fallait fonder notre propre association. (Exemple d'une réponse à la question « racontez brièvement comment est née votre association?»).

Ces associations s'avèrent souvent être des associations «multi-activités » orientant également leurs activités vers la recherche et/ou la prévention sans qu'il soit précisément

\footnotetext{
${ }^{6}$ À partir d'un premier recensement des associations créées entre 1996 et 2005, nous avions procédé en 2006 à une enquête par questionnaire auprès de celles qui étaient comptabilisées comme proposant des activités de soutien aux malades. L'échantillon de base était composé de 248 associations. 30 de ces associations ont été dissoutes, ont arrêté leur activité ou modifié leur objet (ne correspondant plus alors à des activités de soutien aux malades). 33 associations n'ont pas déclaré leur nouvelle adresse. Au total, ce sont donc 185 associations correspondant à la cible qui ont probablement été enquêtées. 42 associations ont retourné le questionnaire complété, ce qui correspond à un taux de réponse de l'ordre de $23 \%$.
} 
possible de savoir quelles sont les activités les plus développées. Soutien moral et psychologique, diffusion d'informations, soins esthétiques, activités culturelles ou aides financières et matérielles sont proposées à destination des patients. Il est à noter qu'aucune des associations enquêtées ne met en avant de quelconques revendications politiques.

La compréhension des formes d'engagement associatif ${ }^{7}$ des patients atteints de cancer nécessite de saisir quelles possibilités sont offertes aux malades de s'investir dans le monde associatif de lutte contre le cancer. En effet, très nombreuses et variées, les associations développent différents types d'activités plus ou moins à même d'être investies par les patients eux-mêmes. De plus, de par leur histoire et leur positionnement sur un véritable «marché » associatif, elles semblent adopter des prises de position différenciées quant au recrutement de bénévoles malades.

\section{Logiques d'engagement dans des associations de lutte contre le cancer}

Pour comprendre les logiques d'engagement des patients ${ }^{8}$ atteints de cancer dans des associations de lutte contre le cancer, nous avons recueilli des données empiriques. L'histoire de l'évolution de l'espace associatif de lutte contre le cancer a orienté le choix des associations à enquêter afin de constituer un échantillon qui rende compte de la diversité de l'offre en ce domaine (tableau 1). Nous avons retenu d'une part, des associations qui historiquement ont été pionnières dans la formalisation d'une aide aux malades et qui se caractérisent par une ancienneté de plus de trente ans et une implantation géographique qui couvre une grande partie du territoire national (par l'intermédiaire de relais à l'échelle du département ou de la région). D'autre part, nous avons cherché à prendre en compte des associations plus jeunes et présentant des actions innovantes. Pour décrire et comprendre au mieux les modes d'organisation des associations ainsi que les modes d'entrée des malades, nous avons cherché à disposer d'une pluralité d'éléments ayant trait autant au fonctionnement formel des associations concernées qu'aux positions et prises de position des différents acteurs associatifs (dirigeants, permanents salariés et bénévoles). Nous avons ainsi récolté un ensemble de données ${ }^{9}$ relatives aux associations enquêtées pour saisir leur position et leurs prises de position à l'égard des malades. Nous avons également conduit des entretiens semidirectifs avec une cinquantaine de membres de ces associations. Ainsi, au minimum cinq personnes ont été interviewées au sein de chacune des associations retenues pour l'étude dont au moins un membre du conseil d'administration.

Au sein des associations investies dans l'aide aux malades, que celles-ci se définissent ou non comme des associations de patients, l'engagement des malades peut être soumis à des critères de sélection plus ou moins formalisés qui vont conditionner, à des degrés divers, la possibilité même de leur engagement ou les rôles susceptibles de leur être dévolus. Une condition que nombre d'associations (dont «Vivre Comme Avant ${ }^{10}$ (VCA) par exemple)

\footnotetext{
${ }^{7}$ Une précision s'impose quant à ce que recouvre le terme d'engagement associatif. Une première distinction s'établit déjà entre bénévole et bénéficiaire. Ce sont les premiers sur lesquels porte notre travail même si les deux s'entremêlent parfois. Une deuxième distinction est également à prendre en compte entre participants et adhérents, notre analyse portant sur les premiers. Ainsi, il s'agit de comprendre l'engagement des «maladesbénévoles-participants ».

${ }^{8}$ Nous utilisons ici le terme «malade » ou «patient atteint de cancer » comme désignant les personnes étant ou ayant été atteintes de cancer, c'est-à-dire qu'elles soient encore en traitement ou considérées en rémission.

${ }^{9}$ Pour chacune des associations enquêtées, nous disposons d'une copie des statuts, d'au moins deux exemplaires (sur la période 2005-2006) du bulletin qu'elles éditent respectivement à l'intention de leurs adhérents et de nombreuses brochures d'information publiées par ces associations.

${ }^{10}$ Vivre Comme Avant est une association animée par des femmes bénévoles qui ont vécu un cancer du sein et qui offrent un soutien moral et matériel aux femmes qui, à leur tour, sont atteintes par cette maladie, notamment dans le cadre de leur prise en charge hospitalière.
} 
posent comme préalable à un engagement des personnes atteintes de cancer est la distance d'avec la maladie mesurée à l'aune de la durée qui les sépare de la fin de leurs traitements. L'idée sous-jacente est d'évaluer le passage du statut de «malade » à celui «d'ancien malade ». L'activité de visite de certaines associations d'entraide tend à justifier un recrutement plus sélectif des malades, dont l'aptitude au bénévolat et le rapport à la maladie ont été évalués positivement. Pourtant, d'autres associations (comme la LNCC) basent leur recrutement sur des principes similaires, défendant l'idée que le recul d'avec la maladie est censé tempérer, d'une certaine manière, l'attitude «agressive» ou «revendicative » de certains patients. Des associations créées plus récemment (comme Jeunes Solidarité Cancer JSC - et Essentielles), et développant notamment des forums de discussion sur Internet, n'adoptent pas ce type de critère de sélection. Au contraire, il y a une volonté explicite de permettre l'engagement quel que soit le moment du parcours de maladie.

Tableau 1 : présentation synthétique des associations enquêtées

\begin{tabular}{|c|c|c|}
\hline Associations & $\begin{array}{l}\text { Année de } \\
\text { création }^{11}\end{array}$ & Objets \\
\hline $\begin{array}{l}\text { LNCC (Ligue nationale } \\
\text { contre le cancer) }\end{array}$ & 1918 & $\begin{array}{l}\text { Aide à la recherche, prévention et information du public, et aide } \\
\text { aux malades. }\end{array}$ \\
\hline $\begin{array}{llr}\text { UAFLMV } & \text { (Union } & \text { des } \\
\text { associations } & \text { françaises } & \text { de } \\
\text { laryngectomisés et mutilés de } \\
\text { la voix) }\end{array}$ & 1958 & $\begin{array}{l}\text { Le soutien, l'entraide, l'incitation à l'apprentissage d'une voix de } \\
\text { remplacement, l'aide morale à la réinsertion et à la réadaptation } \\
\text { familiale, sociale et professionnelle, la défense des intérêts et des } \\
\text { droits des laryngectomisés et autres mutilés de la voix, la vente } \\
\text { d'accessoires et de dispositifs spécifiques. }\end{array}$ \\
\hline VCA (Vivre comme avant) & 1975 & $\begin{array}{l}\text { Répondre, par la réalisation de visites dans les hôpitaux, à l'attente } \\
\text { des femmes opérées ou traitées pour un cancer du sein en leur } \\
\text { proposant le concours d'anciennes patientes qui ont subi et } \\
\text { surmonté des affections analogues. }\end{array}$ \\
\hline $\begin{array}{l}\text { FSF (Fédération des stomisés } \\
\text { de France) }\end{array}$ & 1976 & $\begin{array}{l}\text { Informer et aider à assumer les divers handicaps qu'entraînent la } \\
\text { présence d'une stomie et les exigences de son appareillage, } \\
\text { informer et assister les stomisés et leurs familles pour leur apporter } \\
\text { aide et réconfort, informer les instances administratives } \\
\text { concernées, assurer un réseau d'entraide ou de secours par le biais } \\
\text { de permanences et de contacts, directs ou téléphoniques, } \\
\text { promouvoir activement la prise en charge d'infirmières } \\
\text { spécialisées en stomathérapie, favoriser la réinsertion dans le } \\
\text { monde actif, visiter les futurs ou récents stomisés en pré ou post- } \\
\text { opératoires, soutenir et encourager la prévention. }\end{array}$ \\
\hline $\begin{array}{l}\text { EDFF (Europa Donna Forum } \\
\text { France) }\end{array}$ & 1998 & $\begin{array}{l}\text { Obtenir le soutien et accroître la solidarité des femmes à travers } \\
\text { toute l'Europe face au cancer du sein par : } \\
\text { - } \quad \text { une meilleure formation et information du public et des } \\
\text { professionnels de santé } \\
\text { - } \quad \text { un dépistage ou diagnostic précoce } \\
\text { - } \quad \text { une offre optimisée de soins médico-chirurgicaux et } \\
\text { - psychologiques } \\
\text { - une sensibilisation du public concernant cette maladie } \\
\text { une promotion de la recherche dans ce domaine } \\
\text { particulier de la médecine }\end{array}$ \\
\hline JSC (Jeunes solidarité cancer) & 2002 & Briser l'isolement des jeunes adultes malades, anciens malades et \\
\hline
\end{tabular}

11 Pour les fédérations, souvent constituées dans un deuxième temps, l'année de création correspond aux premières associations créées et appartenant à la fédération. 


\begin{tabular}{|c|c|c|}
\hline & & \begin{tabular}{|l} 
de leurs proches par: \\
- le soutien par le forum de discussion sur Internet \\
- l'information par le site Internet, une série de guides et un \\
carnet de bord \\
- la lutte contre la précarité socioprofessionnelle par un \\
travail de fond en collaboration avec de nombreuses \\
associations.
\end{tabular} \\
\hline Essentielles & 2005 & $\begin{array}{l}\text { Venir en aide aux personnes touchées par le cancer du sein, } \\
\text { faciliter la mise en relation de ces personnes, les informer sur tous } \\
\text { les aspects de la maladie, leur apporter un soutien psychologique } \\
\text { au travers de personnes qui sont ou ont été touchées par la maladie } \\
\text { par la mise à disposition d'un forum de discussion sur Internet. }\end{array}$ \\
\hline
\end{tabular}

Ainsi, élaboré de manière plus ou moins formelle, le recrutement des malades participe à la définition des conceptions de l'engagement légitime des malades développées par chaque association. L'objet de l'association, son histoire mais aussi l'image qu'elle cherche à véhiculer dans l'espace public influencent ses prises de position non seulement quant au possible engagement des malades mais aussi quant à la forme que doit prendre leur encadrement. Ainsi s'opère aussi une distinction, pour ne pas dire une division, entre malades et non-malades. En effet, il ne suffit pas d'avoir été considéré comme malade apte à s'engager pour toujours être en mesure d'occuper des fonctions de responsabilité. Ces formes légitimes d'engagement des malades développées par les dirigeants associatifs - souvent non-malades peuvent donner lieu à des luttes internes quant à leur imposition. Le flou résidant autour des catégories de malades, anciens malades ou non-malades, laisse place à débats.

La manière dont les associations se positionnent les unes par rapport aux autres et formalisent les modes de recrutement et de participation des malades en leur sein contribue à définir les formes que peut revêtir l'engagement associatif des malades. La seule condition d'un diagnostic de cancer ne suffit pas à autoriser l'engagement dans certaines associations où des conditions de recul temporel d'avec la maladie et de modération des revendications sont plus ou moins fortement exigées, contribuant à circonscrire pour les malades l'espace des engagements possibles. Les patients atteints de cancer (même en rémission) n'occupent pas toujours les positions de prise de décision dans les associations (comme par exemple au conseil d'administration), même lorsque le mode de recrutement semble moins sélectif.

Comprendre l'engagement des malades au sein des associations de lutte contre le cancer nécessite certes de circonscrire l'état de l'offre associative, mais il importe aussi de saisir, à partir de l'analyse des parcours singuliers des malades, ce qui caractérise leurs modes d'entrée dans l'espace associatif. Ces modes d'entrée sont à mettre en relation avec le moment de la maladie qui caractérise la personne atteinte de cancer qui s'engage et avec les attentes différenciées qu'il est susceptible d'impliquer.

Un peu plus de la moitié des malades que nous avons interviewés avaient un passé associatif à leur actif avant même leur diagnostic de cancer. Ils semblaient alors reconvertir (Tissot, Gaubert, Lechien, 2005) dans ce domaine un capital associatif, et quelquefois militant, acquis dans d'autres secteurs associatifs. Certains bénévoles ont développé des dispositions au bénévolat qu'ils exprimaient ensuite dans la lutte contre le cancer. Ces dispositions permettent d'expliquer le choix de s'engager dans telle ou telle association du domaine de la lutte contre le cancer proposant des activités similaires à celles précédemment soutenues dans d'autres secteurs associatifs. 
On observe également un recrutement social différencié en fonction des associations. Les bénévoles des associations les plus anciennes étaient majoritairement des femmes inactives, qu'elles soient à la retraite ou sans profession, ayant souvent une ou plusieurs expériences associatives à leur actif avant de s'engager dans la lutte contre le cancer. Seule l'UAFLMV comptait surtout des bénévoles masculins du fait des spécificités épidémiologiques des cancers des voies aérodigestives supérieures. Les associations plus récentes (EDFF, JSC et Essentielles) recrutaient leurs bénévoles parmi les femmes actives exerçant majoritairement des professions intermédiaires. Une part d'affinité élective semblait opérer, homogénéisant le recrutement social des bénévoles au sein de chaque association.

La multiplication des scandales sanitaires au cours des années 1990 et leur médiatisation avec la mise en exergue d'usagers-victimes participent à créer des conditions sociales favorables à la naissance de mouvements contestataires portés par les usagers eux-mêmes, ou par des collectifs parlant en leur nom. Ils parviennent à diffuser auprès d'un très large public, bien au-delà des seules personnes concernées ou susceptibles de l'être, leurs revendications visant au déploiement, en termes de santé publique, de mesures spécifiques de réparation et de prévention. C'est aussi à la fin des années 1990 que vont être créées deux associations de lutte contre le cancer se positionnant clairement du côté d'un activisme politique en faveur des patients. Il s'agit de l'association Europa Donna - Forum France (EDFF) qui tient à constituer un groupe de pression politique spécifiquement dédié à la lutte contre le cancer du sein, et de Jeunes Solidarité Cancer (JSC) qui oriente ses actions de lobbying en direction des adolescents et des jeunes adultes atteints de cancer (tableau 1).

Si ces deux associations se positionnent dans le registre de l'activisme politique et publicisent régulièrement des revendications relatives aux intérêts spécifiques des patients qu'elles représentent, comment les expériences personnelles de la maladie sont-elles publicisées entre les patients eux-mêmes? Le passage d'une expression privée à une publicisation collective va-t-il de soi ? Nous souhaitons ici apporter des éléments de réflexion sur l'articulation entre les domaines sanitaires privés et publics, en mettant plus particulièrement en évidence les difficultés d'une montée en généralité d'histoires singulières.

\section{Registres de publicisation de l'expérience de la maladie}

Afin d'analyser précisément ces difficultés d'articulation entre histoire personnelle et action collective, nous avons procédé, auprès de deux associations - EDFF et JSC -, à des enquêtes de terrain distinctes dont nous reprenons ici les données empiriques. En ce qui concerne EDFF, notre étude portait sur une délégation locale où nous avons réalisé des observations au cours de réunions mensuelles et ce, entre avril 2006 et octobre 2007. D'une durée moyenne de trois heures, ces réunions rassemblaient entre quinze et vingt femmes ${ }^{12}$ considérées comme les membres actives. Nous avons également procédé au recueil d'entretiens semi-directifs avec seize membres de la délégation et deux membres du conseil d'administration de l'association (au niveau national). Parmi les dix-huit personnes interviewées, il y avait quatorze patientes ou anciennes patientes atteintes d'un cancer du sein.

JSC, qui pourtant ne focalise pas ses actions sur un cancer typiquement féminin, ne comptait qu'environ $10 \%$ d'hommes parmi ses membres. Pour cette deuxième association, notre analyse a porté sur les contenus du forum Internet qu'elle propose et ce sur une durée de deux ans (de 2010 à 2012). Soixante-six des cent utilisatrices et utilisateurs répertoriés durant cette période ont indiqué leur âge qui s'élève en moyenne à 26 ans. La tranche d'âge cible de l'association n'est pas clairement délimitée, le terme «jeunes » s'appliquant aussi bien à des

\footnotetext{
12 Ayant spécifiquement pour objet la lutte contre le cancer du sein, les membres de l'association sont
} exclusivement des femmes. 
adolescents, mineurs, qu'à de jeunes adultes voire à des patients plus âgés (l'utilisateur le plus âgé du forum avait quarante-neuf ans). Est «jeune » celui qui se considère comme tel. Nous avons également complété notre enquête par une dizaine d'entretiens semi-directifs réalisés en face-à-face avec des usagers de ce forum et des administrateurs de l'association.

La délégation locale d'EDFF autorisait un engagement associatif dès l'entrée dans la maladie et l'encourageait même dans la mesure où sa création était liée au sentiment d'absence de lieu d'information et d'échanges entre patientes. JSC, de la même manière, grâce à son forum Internet, permettait aux adolescents et aux jeunes adultes de s'exprimer dès l'annonce de leur maladie.

Ainsi, la délégation locale comptait presque exclusivement des patientes ou d'anciennes patientes et souvent il s'agissait de patientes proches du début de leur trajectoire de la maladie (parmi les quatorze patientes interviewées, seules deux avaient pris connaissance de leur diagnostic de cancer du sein (ou de leur récidive) plus de trois ans auparavant). Cette double proximité directe avec la maladie, dans la mesure où les bénévoles étaient ou avaient été ellesmêmes malades et que souvent elles étaient encore en traitement quand elles rejoignaient la délégation locale, transparaît dans les motifs d'engagement exprimés par les bénévoles interviewées.

Cette proximité transparaît également chez les utilisateurs du forum Internet de JSC. Ainsi, parmi les cent utilisateurs du forum recensés entre juin 2010 et juin 2012, quasi les trois quarts étaient des patients en cours de traitement ou d'anciens patients. Et, comme pour la délégation locale d'EDFF, la présentation de son propre parcours revêtait un caractère très important dans la dynamique du forum comme en atteste l'intitulé de la rubrique : "STOP ! Le petit passage obligatoire : tirez-nous votre portrait!». Ainsi, les nouveaux venus sur le forum postaient généralement un premier message dans cette rubrique, détaillant plus ou moins leur parcours de maladie et insistant sur le partage d'une expérience commune.

Bonjour, je suis toute nouvelle sur le forum. J'ai 28 ans et on vient de me diagnostiquer un deuxième cancer du sein... Et oui, après un an et demi (en enfer) a soigné mon sein droit, il y a dix jours on a trouvé un nodule dans mon sein gauche qui s'est avéré cancéreux ! Enfin je suis plutôt bien entourée, il me manque juste l'échange avec des gens de mon âge ayant vécu la même chose, HELP! (message posté en octobre 2011).

L'importance de cette rubrique, de ce passage obligé légitimant en quelque sorte sa présence sur le forum et signifiant l'appartenance à la «classe des malades ${ }^{13}$, s'exprimait également par le nombre de messages postés dans les rubriques à caractère plus personnel (cf. graphique 3).

\footnotetext{
13 Pour Dan Ferrand-Bechmann (2011 : 42), l'appartenance à cette «classe des malades » implique que «les participants s'y sentent épaulés et inclus dans le confort d'une communauté de sentiments, d'émotions et de souvenirs ».
} 
Graphique 3 : Nombre de messages dans les différentes rubriques du forum

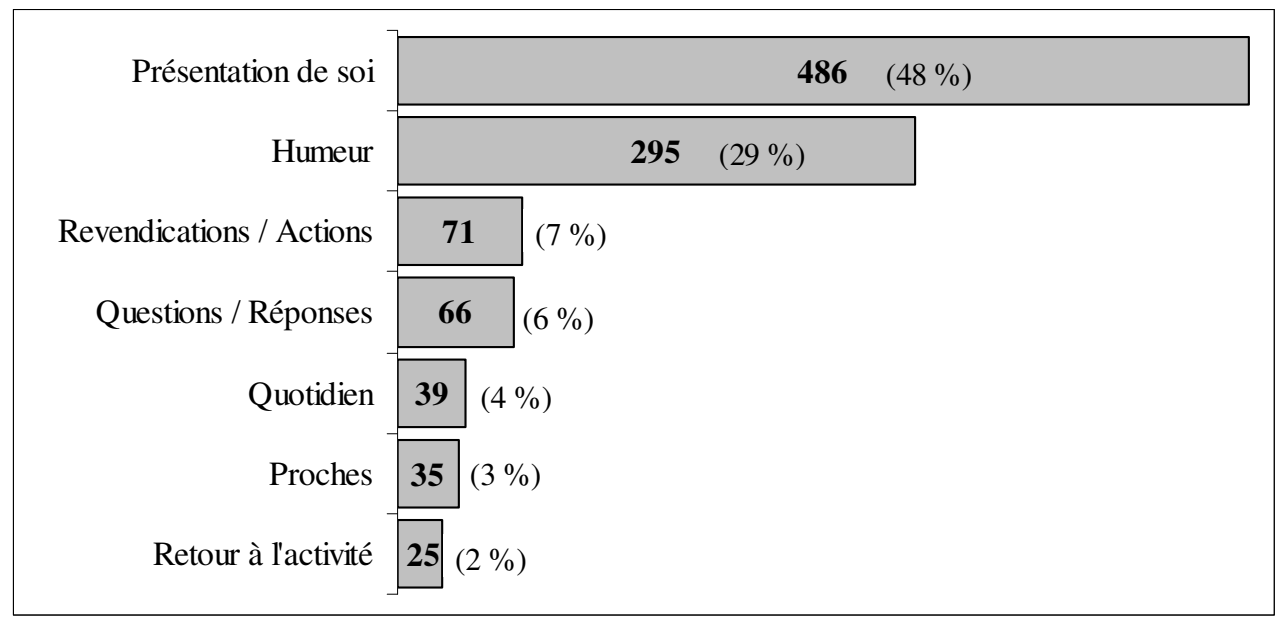

Ce mode de fonctionnement permet certes aux patients et aux anciens patients d'aborder des aspects très intimes liés à leurs traitements ou à leurs angoisses, mais a comme contrepartie une absence fréquente de montée en généralité renforcée par l'idée que «chaque cas est unique ». Il est par conséquent difficile de procéder à des généralisations susceptibles de constituer une cause pouvant faire l'objet d'une mobilisation collective. Faire face, de la sorte, à sa propre maladie ne semble pas favoriser un travail de désingularisation des expériences nécessaire à la montée en généralité et à la formulation de revendications portées au nom d'un collectif (Herzlich, 1995). De manière générale, seuls $9 \%$ des blogs dans le domaine du cancer développent des thèmes relatifs aux politiques de santé (Legros, 2009).

$\mathrm{Au}$ sein de la délégation locale d'EDFF, ce travail de désingularisation semblait être freiné par la manière dont étaient publicisées certaines revendications formulées par les membres de la délégation locale eux-mêmes. En effet, ces revendications prennaient un caractère plus individuel que collectif car elles étaient portées en nom propre et non pas au titre d'un engagement associatif. Leur publicisation n'était pas déléguée à un collectif mais s'appuyait sur un témoignage personnel. La généralisation et l'extrapolation sur lesquelles s'adosse tout travail de construction d'une cause paraissaient alors d'autant plus difficiles à mettre en œuvre.

JSC aussi se positionne comme une association militant pour l'amélioration de la prise en charge spécifique des adolescents et jeunes adultes atteints de cancer. Mais, comme le montre le nombre de messages postés dans les différentes rubriques proposées sur son forum Internet, les messages à caractère revendicatif ou proposant des actions concrètes n'arrivaient qu'en troisième position loin derrière tout ce qui a trait au partage de l'expérience personnelle de la maladie (cf. graphique 3). Et tout comme pour la délégation locale d'EDFF, on constate une oscillation entre préoccupations individuelles et requête collective.

Il semble difficile de dépasser le cas particulier pour évoquer les problèmes posés par le cancer et plus largement les maladies chroniques en termes généralistes. JSC semblait vouloir confier à d'autres organisations le mandat revendicatif et institutionnel. En effet, l'association a opté pour une forme de délégation de cette publicisation en adhérant à d'autres collectifs (comme GoAJA ${ }^{14}$ ) où les malades (ou anciens malades) sont représentés tout en étant associés également à des professionnels de santé qui garantissent probablement une montée en généralité dissociée de la mise en avant de cas particuliers liée, pour les patients, à leur propre

\footnotetext{
${ }^{14}$ Groupe d'Onco-hématologie Adolescents et Jeunes Adultes créé en 2012 à Paris.
} 
vécu. Il en va de même pour EDFF où les administrateurs, donc les interlocuteurs légitimes des diverses institutions ou représentants politiques n'étaient pas d'anciens malades.

Le passage du privé au public ne semble pas aller de soi, sans doute par la difficulté que représente la montée en généralité des expériences singulières des personnes atteintes par la maladie. L'adhésion à une association de lutte contre le cancer, se positionnant de plus comme militante, ne paraît pas suffire à créer les conditions d'une publicisation d'enjeux de santé spécifiques à une pathologie particulière ou plus transversaux en termes de santé publique.

Lorsque les patients ou anciens patients atteints de cancer prennent la parole, il s'agit la plupart du temps, comme nous l'avons vu à travers l'exemple de deux associations à vocation militante, d'une publicisation entre-soi. Ils parlent de leur maladie, de leur parcours, de leurs problèmes, de leurs satisfactions et insatisfactions dans un cadre circonscrit aux réunions de l'association (pour la délégation locale) ou au forum Internet (pour JSC). Le régime de proximité directe avec la maladie, lié au fait d'être ou d'avoir été soi-même malade et de souvent être proche du début du parcours de maladie lors de l'engagement associatif, renforce la valorisation de l'entraide et du partage d'expériences. Cette proximité semble surtout valoriser une prise de parole individuelle soulignant la particularité de chaque expérience et s'inscrit dans une temporalité circonscrite à une période donnée ce que confirme le renouvellement régulier des bénévoles :

Le truc avec JSC quand même, ça fait trois ans que j'y suis, je l'ai vu, c'est qu'il y a un turn-over énorme de bénévoles. Entre les bénévoles qu'il y avait quand je suis arrivée et ceux qu'il y a aujourd'hui, il n'y a pratiquement plus les mêmes. Donc moi, j'avais créé des liens avec des anciens, les trois-quatre je ne les vois plus dans JSC. Pourquoi ? Parce que c'est des gens investis plus jeunes que moi, qui ont eu [leur cancer] plus jeune, qui n'avait pas forcément..., dont deux par exemple, elles viennent d'avoir des bébés, donc elles sont dans une autre construction de vie. (...) Il y a beaucoup de jeunes qui s'investissent à un moment donné, mais après la vie reprends le dessus et tant mieux et donc ils priorisent sur leur boulot, leur vie de famille etc. (femme, 34 ans, membre de JSC depuis 2009).

L'événement que représente la survenue d'un cancer dans la trajectoire biographique d'un individu, même s'il n'entraîne pas nécessairement une rupture biographique (Bury, 1982 ; Voegtli, 2004), représente un accident de la vie qui a indéniablement des conséquences sur la vie quotidienne : diminution des relations sociales voire isolement, atteintes physiques momentanées ou durables, abandon ou transformations d'un ensemble d'activités, etc. obligeant à un réaménagement du quotidien (Knobé, Marsault, 2014). Face à cette épreuve, la recherche d'un entre-soi, de liens sociaux entre « frères d'armes » d'une certaine manière, se fonde sur une proximité émotionnelle liée aux ressentis engendrés par la maladie et circonscrite à une temporalité précise. Si dans un premier temps, la proximité avec la maladie semble freiner une forme publique de publicisation, une certaine prise de distance en cas de trajectoire ascendante paraît la décourager à son tour. La trajectoire de la maladie (Ménoret, 1999) influe sans conteste sur les attentes relatives à l'engagement associatif (Knobé, 2007 ; Ferrand-Bechmann, 2011).

\section{Conclusion}

Les analyses présentées ici apportent des éléments de compréhension des grandes tendances de création et de développement des associations de lutte contre le cancer en France, et permettent un focus sur les difficultés à la politisation des discours des patients eux-mêmes à travers l'exemple de deux associations à vocation militante. La délicate conciliation entre intérêts personnels et cause collective, à l'échelle locale d'une association, freine la formalisation de revendications portées par les patients. Ce constat ne doit pas 
occulter le fait que les mobilisations associatives en France, comme dans d'autres pays (Briatte, 2008), ont encouragé la mise à l'agenda politique de la lutte contre le cancer (Ravier, 2007). Bien qu'elle soit fortement polarisée autour d'enjeux biomédicaux (Vézian, 2014), les patients semblent avoir fait entendre leurs voix, souvent par l'intermédiaire de porte-parole.

Au-delà de la lutte contre le cancer, la représentation des usagers de la santé s'est en effet institutionnalisée en France au cours des années 1990 notamment par le travail militant effectué par le Collectif interassociatif sur la santé (CISS) (Knobé, 2010) devenu France Assos Santé en mars $2017^{15}$. L'efficacité de cette publicisation tient à l'alliance des plus grandes associations françaises dans le domaine des luttes contre les maladies (dont La ligue nationale contre le cancer, AIDES, l'association française contre les myopathies, etc.) sur des thématiques transversales de santé publique. La montée en généralité a consisté pour ce collectif d'associations a dépassé les intérêts particuliers liés à chaque pathologie spécifique pour penser et revendiquer des enjeux plus globaux et transversaux (relatifs aux droits des malades ou à l'accès aux soins par exemple). Si des risques d'instrumentalisation de la représentation des patients persistent, son institutionnalisation et sa reconnaissance par l'État permettent une expression de leurs revendications.

\section{Références bibliographiques}

Barbot J. (2002), Les malades en mouvements. La médecine et la science à l'épreuve du sida, Paris, Balland.

Barbot J., Fillion E. (2007), «La dynamique des victimes. Les formes d'engagement associatif face aux contaminations iatrogènes [VIH et prion] », Sociologie et Sociétés, $\mathrm{n}^{\circ} 1$, p. $217-247$.

Bevetty F., Dufranc C., Hofmann G. (2006), « Critères de qualité de l'annonce du diagnostic : point de vue des malades et de la Ligue nationale contre le cancer », Risques et Qualité, $\mathrm{n}^{\circ} 2$, p. 67-72.

Briatte F. (2008), « Policy Involvement among Executive Leaders: Cancer Control in England and France, 1997-2007 », Political Studies Association (PSA) Northern Postgraduate Conference, Edinburgh, United Kingdom.

Briatte F., Knobé S. (2010), «Institutionnalisation et médiatisation d'une prise de parole collective chez les malades atteints de cancer: les "États généraux" de la Ligue nationale contre le cancer », in H. Romeyer (dir), Communication, médias, discours et santé, Éd. EHESP, Rennes, p. 159-173.

Bury M. (1982), «Chronic illness as biographical disruption », Sociology of health and illness, $\mathrm{n}^{\circ} 4$, p. 168-182.

Derbez B., Rollin Z. (2016), Sociologie du cancer, Paris, La Découverte.

Dodier N. (2003), Leçons politiques de l'épidémie de sida, Paris, Éditions de l'École des Hautes Études en Sciences Sociales.

Duchesne S., Haegel F. (2001), «Entretiens dans la cité, ou comment la parole se politise », EspacesTemps, ${ }^{\circ} 76 / 77$, p. 95-109.

\footnotetext{
${ }^{15}$ Décret n ${ }^{\circ}$ 2017-90 du 26 janvier 2017 relatif à l'Union nationale des associations agréées d'usagers du système de santé, paru au Journal officiel n ${ }^{\circ} 0024$ du 28 janvier 2017. Le CISS créé en 1996 comptait 15 associations membres, France Assos Santé en compte 76 en 2018.
} 
Duchesne S., Haegel F. (2004), «La politisation des discussions, au croisement des logiques de spécialisation et de conflictualisation », Revue française de science politique, $\mathrm{n}^{\circ} 6$, p. 877-909.

Eliasoph N. (1998), Avoidings politics, Cambridge, Cambridge University Press.

Ferrand-Bechmann D. (2011), Les bénévoles face au cancer, Paris, Desclée de Brouwer.

Fillion E. (2009), À l'épreuve du sang contaminé : Pour une sociologie des affaires médicales, Paris, Éditions de l'École des Hautes Études en Sciences Sociales.

Gaudillière J-P. (2002), « Mettre les savoirs en débat ? Expertise biomédicale et mobilisations associatives aux États-Unis et en France », Politix, n57, p. 103-122.

Hamidi C. (2006), «Éléments pour une approche interactionniste de la politisation. Engagement associatif et rapport au politique dans des associations locales issues de l'immigration », Revue française de science politique, ${ }^{\circ} 1$, p. 5-25.

Henry E. (2000), Un scandale improbable. Amiante: d'une maladie professionnelle à une "crise de santé publique », Thèse de doctorat en sciences de l'information et de la communication, Université de technologie de Compiègne.

Herzlich C. (1995), «Les difficultés de constitution d'une cause », Sciences sociales et santé, $\mathrm{n}^{\circ} 4$, p. 39-44.

Hirsch M., Duneton P., Baralon P., Noiville F. (1996), L'affolante histoire de la vache folle, Paris, Balland.

Knobé S. (2007), Le besoin d'inscription dans des collectifs de patients atteints de cancer participation associative, structuration des collectifs de malades, Rapport de postdoctorat pour l'Institut national du cancer.

Knobé S. (2009), «Logiques d'engagement des malades dans les associations de lutte contre le cancer», Socio-logos, $n^{\circ} 4$, [en ligne] : www.socio-logos.revues.org.

Knobé S. (2010), «À propos des collectifs d'usagers de la santé en France au $20^{\text {ème }}$ siècle », Journal of global health promotion, $\mathrm{n}^{\circ} 3$, p. 85-88.

Knobé S. (2011), «Parcours et interactions associatives en contexte de lutte contre le cancer du sein. Des freins à la politisation des discours », Sociologie Santé, n³3, p. 253-272.

Knobé S. (2015), «Une publicisation entre-soi : quand les patients atteints de cancer prennent la parole », in A. Meidani, E. Legrand, et B. Jacques (dir.), Du public au privé : une affaire de santé, Éd. EHESP, Rennes, p. 69-81.

Knobé S., Marsault C. (2014), «La pratique sportive comme indicateur de santé ? L'exemple des adolescents atteints de cancer », in C. Marsault, S. Cornus (dir.), Santé et EPS. Un prétexte, des réalités, L'Harmattan, Paris, p. 129-140.

La Ligue nationale contre le cancer (1999), Les malades prennent la parole. Le livre blanc des $1^{\text {ers }}$ États Généraux des malades du cancer, Paris, Ramsay.

Legros M. (2009), «Étude exploratoire sur les blogs personnels santé et maladie », Santé publique, vol. 21, HS nov.-dec., p. 41-51.

Mayer N. (dir.) (2000), Dynamique de l'engagement et élargissement des solidarités, Rapport final de l'appel d'offres de recherche MIRE, Fondation de France, Crédit coopératif «Produire les solidarités ».

Ménoret M. (1999), Les temps du cancer, Paris, Éditions du CNRS. 
Ménoret M. (2002), «Innovation génétique et cancer du sein : l'organisation des rapports entre professionnels et usagers en France et aux États-Unis », Innovations et sociétés, $\mathrm{n}^{\circ} 2$, p. 37-54.

Ménoret M. (2006), «Prévention du cancer du sein : cachez ce politique que je ne saurais voir », Nouvelles questions féministes, $\mathrm{n}^{\circ} 2$, p. 32-47.

Mougeot F., Robelet M., Rambaud C., Occelli P., Buchet-Poyau K., Touzet S., Philippe Michel Ph. (2018), «L'émergence du patient-acteur dans la sécurité des soins en France : une revue narrative de la littérature entre sciences sociales et santé publique », Santé Publique, $\mathrm{n}^{\circ} 1, \mathrm{p} .73-81$.

Pinell P. (1992), Naissance d'un fléau. Histoire de la lutte contre le cancer (1890-1940), Paris, Métailié.

Pinell P. (1997), «Pour une étude du mouvement associatif. Éléments d'analyse sociologique », Gestions Hospitalières, n³68, p. 498-504.

Pinell P. (2002), Une épidémie politique : la lutte contre le sida en France (1981-1996), Paris : PUF.

Pinell P. (2007), «Éléments pour une réflexion sociologique sur le phénomène associatif dans le domaine de la santé », Sociologie Santé, n²6, p. 19-30.

Pollak M. (1991), «Constitution, diversification et échec de la généralisation d'une grande cause », Politix, n 16, p. 80-90.

Ravier M. (2007), La construction d'un problème public : la lutte contre le cancer en France (1945-2000), Mémoire de master de sciences politiques, IEP de Lyon.

Tabuteau D. (2007), Les droits des malades et des usagers du système de santé, une législature plus tard, Paris, Éditions de Santé/Presses de Sciences Po.

Tabuteau D. (2013), Démocratie sanitaire : les nouveaux défis de la politique de santé, Paris, Odile Jacob.

Tissot S., Gaubert C., Lechien M-H. (2005) (coord.), Reconversions militantes, Limoges, Presses Universitaires de Limoges.

Veil J-F. (1998), La santé publique atomisée. Radioactivité et leucémies : les leçons de la Hague, Paris, La Découverte.

Vézian A. (2014), «À la recherche d'une politique biomédicale en France : chronique d'une réforme inaboutie en cancérologie », Sociologie du travail, n² 2, p. 204-224.

Vilain J-P., Lemieux C. (1998), «La mobilisation des victimes d'accidents collectifs. Vers la notion de "groupe circonstanciel" », Politix, n44, p. 135-160.

Voegtli M. (2004), «Du Jeu dans le Je: ruptures biographiques et travail de mise en cohérence », Lien social et politiques - RIAC, n51, p. 145-158. 